CAHIERS DE

NARRATOLOGIE

\section{Cahiers de Narratologie}

Analyse et théorie narratives

31 Bis | 2017

Espace du récit, récit de l'espace en contexte germanique

\title{
La Bibliothèque nationale de France et le camp Austerlitz. La narration d'un espace traumatisé chez Sebald
}

Judith Kasper

Translator. l'allemand par Emmanuel Faure

\section{(2) OpenEdition}

\section{Journals}

Electronic version

URL: http://journals.openedition.org/narratologie/7675

DOI: 10.4000/narratologie.7675

ISSN: 1765-307X

Publisher

LIRCES

\section{Electronic reference}

Judith Kasper, «La Bibliothèque nationale de France et le camp Austerlitz. La narration d'un espace traumatisé chez Sebald », Cahiers de Narratologie [Online], 31 Bis | 2017, Online since 26 June 2017, connection on 01 May 2019. URL : http://journals.openedition.org/narratologie/7675; DOI : 10.4000/ narratologie. 7675

This text was automatically generated on 1 May 2019.

Article L.111-1 du Code de la propriété intellectuelle. 


\title{
La Bibliothèque nationale de France et le camp Austerlitz. La narration d'un espace traumatisé chez Sebald
}

\author{
Judith Kasper \\ Translation : I'allemand par Emmanuel Faure
}

\section{AUTHOR'S NOTE}

Cet article est, sauf quelques modifications et actualisations, la traduction de l'essai suivant: Judith Kasper, « Die Bibliothèque Nationale de France und das Phantasma eines Lagers », dans Die Transformation der Orte. Annäherung an die nationalsozialistischen Konzentrations- und Vernichtungslager, éd. Par Alexandra Klei, Katrin Stoll et Annika Wienert, Bielefeld, transkript, 2011, S. 101-118.

\section{Images fixes et déplacements}

Faire de l'histoire [...], ce n'[est] que s'intéresser à des images préétablies, ancrées à l'intérieur de nos têtes, sur lesquelles nous gardons le regard fixé tandis que la vérité se trouve ailleurs, quelque part à l'écart, en un lieu que personne n'a encore découvert ${ }^{1}$.

Ces lignes sont extraites du roman Austerlitz de W. G. Sebald, dont la version originale allemande est parue en $2001^{2}$.

2 À première vue, ces propos pourraient passer pour un rejet de l'historiographie. Préjugés, stéréotypes et fantasmes, c'est ce que suggère la citation, déterminent le regard posé sur l'histoire et mènent inévitablement à des déformations. Et pourtant, cette affirmation figure dans une œuvre littéraire qui explore d'une manière presque inégalable les traces laissées par l'histoire dans notre époque actuelle - et notamment les conséquences catastrophiques de la politique d'extermination national-socialiste. Les paroles sont celles du protagoniste, Austerlitz, qui s'engage corps et âme pour retrouver ce lieu «à l'écart » 
de l'histoire, encore inexploré. Ainsi, ces lignes ne sont pas une simple condamnation, elles introduisent bien plutôt un travail spécifique sur les "images préétablies", " ancrées ", un relâchement et un déplacement du regard de ces images vers d'autres, sans toutefois jamais échapper véritablement - précisons-le d'emblée - aux fantasmes suscités par l'histoire, sans jamais découvrir ce «lieu [à l'écart] que personne n'a découvert ", ni pouvoir le transférer vers un hic et nunc saisissable. L'enjeu est cependant de parcourir l'espace intermédiaire, au-delà des images préétablies et en-deçà du lieu à l'écart, inaccessible. L'enjeu est, en partant de cette observation critique, de dynamiser les images de l'histoire, en particulier celles du camp de concentration.

3 Si l'on voulait résumer en un mot l'image du camp de concentration " ancrée dans nos têtes ", on pourrait peut-être le faire de la façon suivante: ce qui domine, c'est la représentation du camp comme " cosmos verrouillé » hermétiquement, qui a reçu à une date relativement récente une caution scientifique dans le livre de Wolfgang Sofsky, Ordnung des Terrors ${ }^{3}$. Ce « cosmos verrouillé ", pourrait-on ajouter, se trouve "quelque part à l'écart », très loin. Dans les témoignages des survivants, on a fréquemment l'impression d'un voyage interminable, effectué dans des conditions extrêmes pour rejoindre un lointain territoire inconnu. Au fond, le «cosmos verrouillé » est pensé comme une grande prison. Toutefois, ces «images fixes » méconnaissent totalement la réalité des camps, comme l'explique Primo Levi dans son recueil d'essais I sommersi e i salvati (1986), dans lequel il se confronte explicitement aux stéréotypes dont fourmille la mémoire du camp. Levi est pleinement conscient que ces stéréotypes - par exemple la représentation du camp comme une prison dont on peut s'échapper pour peu qu'on soit suffisamment débrouillard ${ }^{4}$ - ne peuvent être purement et simplement éliminés et sont eux-mêmes une séquelle de la blessure, c'est-à-dire du traumatisme qui a marqué irréparablement la mémoire des survivants et aussi celle de la postérité en général : le souvenir du camp, sa représentation linguistique et visuelle, est donc nécessairement falsifiés.

4 Il est caractéristique que Levi lui-même soit constamment obligé de recourir à des formes stéréotypées de témoignage. Ainsi est-il prié par une classe de primaire de tracer au tableau le plan du camp, y compris les miradors, les portes, les réseaux de barbelés et la centrale électrique. Il s'exécute, non sans gêne. Un élève lui explique alors, sur la base du croquis, comment il aurait pu s'évader. Levi décrit cette scène en détail et en tire la conclusion que le fossé séparant les faits et l'image qu'en donnent les livres, les films et autres récits ne cesse de s'élargir'.

5 L'éloignement des camps, leur isolement «à l'écart » doit ainsi être compris dans un sens moins spatial que mental et langagier. Le degré de convergence de ces deux plans se révèle si l'on garde à l'esprit les mystifications langagières des national-socialistes. Au lieu de "déportation à Auschwitz, Birkenau ou Treblinka ", ils parlent de "départs à l'étranger ", d' 'évacuations vers l'Est " ${ }^{7}$. Rapprocher les camps de notre présent implique donc toujours un travail sur le langage et les images, par le biais desquels le camp s'est manifesté dans notre mémoire. En ce sens, la transformation des camps désigne l'irruption du camp dans l'image, où il se fige, puis les métamorphoses de cette image, elles-mêmes observables à deux niveaux: d'une part comme persistance tenace de l'image fixe, résultant d'une sorte de compulsion de répétition ; mais aussi comme travail critique sur cette image, comme tentative de rompre avec des représentations figées en les dynamisant. 
6 De même que l'inconscient, inaccessible et isolé, peut se manifester inopinément dans le présent d'un acte manqué langagier, de même la réalité lointaine du camp apparaît par moments toute proche, par exemple en plein milieu des villes dans lesquelles nous vivons et travaillons aujourd'hui. Ces derniers temps, cet état de faits suscite l'intérêt croissant des historiens ${ }^{8}$. La longue latence qui a précédé la rencontre du regard scientifique avec cette proximité étrange et mystérieuse est sans doute due en partie à la mystification qui a entouré dès le début les camps nazis et qui continue à marquer les témoignages et également la science. Les conventions langagières, inséparables de l'extermination ellemême, ont eu pour résultat la persistance, jusqu'à aujourd'hui, des résistances mentales à l'identification de l'extermination, non seulement dans son extension spatiale, mais aussi dans sa transgression conceptuelle. Le transfert qui s'observe dans les recherches historiques les plus récentes vers les multiples entrelacements de la réalité concentrationnaire avec le domaine de la vie civile peut être vu comme un travail sur ces résistances mentales. Ce sont notamment des œuvres littéraires qui ont sensiblement contribué à la dynamisation des images fixes du camp9. Toutefois, souvent la communication entre les divers systèmes discursifs - histoire, études culturelles, recherche littéraire, témoignages, textes littéraires - reste balbutiante, du fait notamment des limites dans lesquelles restent confinées la structuration de notre savoir et son évolution au sein de chaque discipline.

7 D'une manière en quelque sorte paradigmatique, ces limites étroites faisant résolument obstacle à toute inter- et transdisciplinarité sont représentées par la nouvelle Bibliothèque nationale de France, à Paris. "Toute la mémoire du monde ${ }^{10}$ » y est subdivisée en quatre domaines ayant également donné leur nom aux quatre tours emblématiques de la bibliothèque, qui figurent symboliquement quatre livres ouverts : tour des Temps, tour des Lois, tour des Nombres, tour des Lettres. On conserve là tout le savoir du monde, tel est l'objectif revendiqué, tout en le soumettant à une division spatiale et systématique si nette que le travail des visiteurs s'en ressent. Ainsi, il est compliqué sinon interdit aux lecteurs d'emporter un livre de la tour des Temps dans celle des Lettres, et relier les nombres aux lois, les lois au temps et aux lettres requiert pour ainsi dire une transgression institutionnelle.

\section{Une bibliothèque à l'ombre d'un ancien camp}

8 Avec la nouvelle Bibliothèque nationale de France, solennellement inaugurée en 1995 et ouverte au public en 1998, se concrétise en partie ce lieu historique «à l'écart» mentionné dans l'extrait cité au début de cet article :

Sur le terrain vague où s'élève aujourd'hui cette bibliothèque, délimité par le triage de la gare d'Austerlitz et le pont de Tolbiac, il y avait par exemple jusqu'à la fin de la guerre un vaste entrepôt où les Allemands regroupaient tous les biens pillés dans les appartements des Juifs parisiens ${ }^{11}$.

Un entrepôt, voire un camp (Lager), est localisé là où se trouvait dans l'intervalle un «terrain vague ", pour ainsi dire un "néant» demandant à être comblé et utilisé, a fortiori dans la densité de l'espace urbain parisien. Le «terrain vague » n'est cependant qu'une strate intermédiaire venue se glisser entre le camp et la bibliothèque. Elle sépare les deux réalités là où elles menacent d'entrer directement en collision. La phrase littéraire réunit désormais ce qui n'est aujourd'hui problématisé nulle part à cet endroit ${ }^{12}$. 
10 Cette phrase est donc le résultat d'une série de déplacements et de condensations. Elle provoque en outre un déplacement supplémentaire, dans la représentation du lecteur qui connaît peu ou prou l'histoire officielle des camps en France, puisqu'aux côtés du camp bien connu de Drancy, dans la banlieue nord-est de Paris, apparaît désormais un autre camp, en grande partie inconnu. D'un coup, la représentation du camp fait irruption dans Paris intra-muros, et inversement, Paris intra-muros est hanté par le camp ${ }^{13}$.

11 Ce qui est exprimé par cette phrase fictionnelle - au sens de "phrase figurant dans un texte fictionnel », ce qui n'en fait pas pour autant une phrase fictive - remonte à un texte non-fictionnel, plus précisément à l'article « Die Türme des Schweigens » [Les tours du silence], publié à l'hiver 1996/97 par le journaliste Alexander Smoltczyk dans un numéro du magazine de l'hebdomadaire Die Zeit ${ }^{14}$. On peut y lire :

Il y a un mois a été inaugurée en grande pompe la nouvelle Bibliothèque nationale de France. À l'emplacement d'un terrain vague, dit-on. En réalité, la plus grande réserve de savoir d'Europe s'élève sur un lieu marqué par la barbarie : dès 1943, on $\mathrm{y}$ a concentré des bibliothèques, dont les propriétaires juifs avaient été spoliés. Mais de cela, on ne parle pas $^{15}$.

Et plus loin :

En novembre 1943, les SS ont ouvert le camp «Austerlitz» sur le terrain des installations ferroviaires de Tolbiac. C'était une annexe du camp de concentration de Drancy. [...] À proximité directe de l'emplacement de la nouvelle bibliothèque, aux magasins généraux du 43, quai de la Gare (rebaptisé quai Panhard-et-Levassor) jusqu'en août 1944, les biens juifs ont été triés [par des "demi-Juifs », en jargon nazi, ou des Juifs "conjoints d'aryen»] pour être envoyés dans les villes bombardées du Reich ${ }^{16}$.

Cet article de journal bien documenté a rencontré relativement peu d'écho, à l'exception des articles du Monde et de Libération mentionnés ci-dessus (note 12). Smoltczyk n'est cependant pas le premier à s'être intéressé à cette constellation : des artistes établis dans le squat des Frigos, à l'emplacement des anciens entrepôts frigorifiques de la ville essaient eux-mêmes depuis des années - en vain - d'attirer l'attention sur la coïncidence inouïe qui superpose aujourd'hui le symbole par excellence de la mémoire culturelle et nationale, la nouvelle Bibliothèque nationale de France, et l'ancienne annexe du camp d'internement de Drancy. La mémoire culturelle et nationale n'a pas encore intégré cette superposition ; quand elle est évoquée, c'est de façon ponctuelle, sans donc provoquer de " réaction adéquate ${ }^{17}$ ", pour parler en termes freudiens, comme si ce fait résistait d'une certaine manière à la prise de conscience. On pourrait faire l'hypothèse suivante : c'est précisément parce que ce fait résiste à la conscience et n'est pas intégrable à la mémoire culturelle qu'il se manifeste dans un texte littéraire. Une fois stocké à cet endroit, il participe à la mémoire culturelle sans perturber l'intention symbolique de l'expression architecturale de la bibliothèque. On se trouve ainsi en présence d'une forme de compromis, résultat typique de la condensation et du déplacement ${ }^{18}$.

L'élaboration d'un compromis non seulement institutionnel, mais aussi langagier, peut être mise en évidence par l'analyse des discours officiels portant sur la bibliothèque. On commencera par citer l'architecte Dominique Perrault, qui déclare à propos de son œuvre :

C'était un énoncé manifeste toujours d'actualité et qui résume bien le projet : « une place pour Paris, une bibliothèque pour la France ». [...] Nous avions présenté des planches de dessins montrant comment les monuments se positionnaient accompagnés de grands vides, le long du fleuve : nous évoquions la relation du Champ-de-Mars, des Invalides, des Tuileries, du Jardin des Plantes, avec la Seine. 
Ces références nous permettaient de développer l'idée de rééquilibrer Paris vers l'est par l'inscription de grands équipements publics ou de grands jardins. Jusqu'alors, le ministère des Finances fonctionnait comme une barrière d'octroi qui, une fois dépassée, donnait l'impression de ne plus être à Paris. Ce que nous voulions, a contrario, c'était dilater Paris jusqu'au boulevard périphérique. [...] Ces «quatre livres ouverts» énoncent clairement un signe. [...] [C]'est comme un moyen mnémotechnique pour identifier et repérer la bibliothèque dans la ville. Ensuite, d'autres perceptions moins immédiates, plus contradictoires et complexes, apporteront d'autres niveaux de lecture. [...] J'ai cherché dans la bibliothèque à retrouver des émotions bâties sur des paradoxes entre présence et absence, entre monumental et humain, entre opaque et lumineux, sans parler d'être ou de ne pas être dans Paris. [...] L'architecture de la Bibliothèque est composée de ces matières abstraites que sont le vide et le silence. [...] Ce monument trace un acte fondateur pour l'Est parisien. Ce n'est pas quelque chose de planté là, seul ; c'est un lieu qui se découvre, un lieu initiatique qui doit être vécu pour être compris. La virtualité de cet espace introduit la notion d'abstraction en architecture. Sur l'esplanade piétonne, prise entre les quatre angles et au bord du jardin central, les systèmes d'absence, d'inversion, de cadrage trouvent une autre évidence. [...] Elle [l'architecture] est le sismographe de l'époque et rend compte de notre culture ${ }^{19}$.

Comme le montre clairement cette longue citation, la rhétorique employée cherche à gagner le lecteur à une cause vis-à-vis de laquelle il pourrait manifestement avoir des doutes $^{20}$. À première vue, le discours entend convaincre que le chantier en cours, chargé de prestige, apporte en outre l'idée nationale et culturelle française dans un espace urbain parisien jusque là sous-développé de ce point de vue, contribuant ainsi efficacement à la «rédemption du lieu ${ }^{21} »$; ce même discours inverse toutefois ses prémisses si nous le faisons précéder de la superposition factuelle du camp et de la bibliothèque, ignorée par le discours lui-même. Si l'on prend au mot l'injonction faite aux critiques d'art - Perrault se targuant de suggérer lui-même "d'autres niveaux » de lecture du symbolisme architectural - les notions-clés que constituent «le vide», «le silence ", « l'absence ", « l'inversion », « être ou ne pas être à Paris » ouvrent un champ de connotations renvoyant justement à ce qui n'est pas dit ici.

Remarquons d'ailleurs qu'il n'est pas question ici de la réalité historique du camp d'Austerlitz, cette annexe de Drancy où, à partir de juillet 1943, les biens spoliés des Juifs parisiens déportés furent réparés, récupérés et préparés pour être envoyés dans les villes allemandes ${ }^{22}$. Bien plutôt, la représentation d'un camp émerge du discours comme l'image ambiguë d'un portrait caché. On observe un phénomène similaire dans les discours des critiques de la bibliothèque. On citera un texte paru dans la revue Le Débat, dont les prestigieuses colonnes ont abrité en 1999/2000 une virulente polémique contre un bâtiment qui semblait essentiellement motivé par des considérations de prestige. Pour les contributeurs, la nouvelle Bibliothèque nationale est une "honte nationale", un "délire pharaonique ", un " héritage encombrant", une "humiliation nationale ", une "bibliothèque maudite», un "tombeau pour le livre», une "malédiction», une " catastrophe ${ }^{23} »$.

17 L'historien français Pierre Nora, rendu célèbre dès le milieu des années 1980 par le concept de « lieu de mémoire» (et fondateur de la revue), a été l'un des protagonistes de cette attaque en règle contre la bibliothèque. L'un des textes qu'il a publiés dans le cadre de cette polémique est intitulé «Retour sur les lieux du crime». Mais le lecteur qui s'attendrait à y trouver des réflexions sur la problématique du lieu et son passé d'ancien camp nazi sera déçu: Nora n'évoque nullement la présence antérieure d'un camp à 
l'emplacement de la bibliothèque, il esquisse au contraire un état des lieux démoniaque du camp que constituerait la bibliothèque elle-même :

L'historique des malheurs qu'a connus la B.N.F. [...] n'est plus à faire. [...] Malheureusement, il est clair que l'institution ne se remettra pas de si tôt de ses contradictions initiales et de ses erreurs de principe. [...] Les malformations de naissance qu'on découvre aujourd'hui paraitront d'ailleurs d'autant moins supportables que les objectifs [...] sont en train d'être revus à la baisse [...]. L'erreur de base est inscrite dans le projet lui-même. Un bon sens élémentaire aurait notamment suggéré que la bibliothèque consiste dans une salle de lecture centrale vers laquelle puissent converger les livres. Non : une esplanade vide de six hectares - de surcroit plantée d'arbres qui interdisent la construction de magasins souterrains. Un rectangle inutile flanqué de quatre tours d'angle distantes de près de trois cents mètres, qui condamnent à des circulations permanentes et épuisantes. [...] L'impression prévaut d'un programme pervers méthodiquement poursuivi et tenacement appliqué. Prenez l'escalier: on ne le monte dans son inutile majesté que pour redescendre, comme dans un puits, par un vertigineux trottoir roulant. Ce génie du contraste et de la disproportion se vérifie dans tous les éléments essentiels de la bibliothèque. [...] Exiguïté, courants d'air, architecture brute, postes de travail installés de façon invraisemblable [...], éclairage souvent inadapté, morcellement des tâches, espace confiné, traité à l'économie. Même contraste et même disproportion entre ces couloirs qui n'en finissent pas et ces sas métallisés dont on se sent prisonnier, ces portes de prison, dix mille au total, qui font qu'on ne sort de l'agoraphobie que pour tomber dans la claustrophobie ${ }^{24}$.

On pourrait y ajouter les innombrables comptes-rendus de visiteurs relatant leur expérience de la bibliothèque. Partout s'impose un vocabulaire qui ne peut que paraître inadéquat au regard d'une série de défauts techniques et de maladresses architecturales. La surdétermination des mots s'explique peut-être rationnellement par le fossé entre les ambitions démesurées entachant le projet et son exécution défectueuse. On retiendra néanmoins que les termes choisis pour exprimer ce fossé renferment un potentiel sémantique où transparaît constamment le stéréotype fantasmagorique d'un camp.

\section{Fantasme - traumatisme - exception}

19 Dans une perspective psychanalytique, un fantasme se forme à la place d'un souvenir lorsque ce souvenir, processus dynamique toujours ouvert, est remplacé par une image qui prétend représenter un évènement comme un état isolé et révolu. Pour le camp, l'isolement temporel s'illustre par la représentation d'un isolement spatial. Mais le simple fait que l'image ainsi close - les quatre miradors avec des barbelés que Levi dut dessiner au tableau - se retrouve telle quelle dans les diverses descriptions de la bibliothèque atteste que nous avons précisément affaire à un aspect de l'histoire qui n'est ni clos, ni isolé. Car l'image stéréotypée se révèle être un fantasme dès lors qu'elle s'impose inconsciemment et inopportunément en présence d'un énoncé visant manifestement un tout autre domaine de référence. Or, les fantasmes se forment en priorité à la suite d'évènements traumatisants. Sont traumatisants les évènements qui, lors d'un choc psychique, entraînent une blessure si profonde que la psyché n'est plus en mesure de réagir de manière appropriée. L'absence de "réaction adéquate » au moment du choc - mais aussi plus tard - peut avoir des causes différentes. Outre l'aspect temporel du trauma, Freud mentionne d'autres possibilités, comme la honte, les tabous sociaux, ou encore la répression intentionnelle d'une pensée embarrassante ${ }^{25}$. À la manière d'un fantôme, dont il tire son nom, le fantasme se substituant au souvenir hante tel lieu, puis 
tel autre, toujours insaisissable. Fantôme, il forme un corps étranger qui ne peut s'intégrer à aucune image, mais participe pour une large part à la formation d'images, de représentations.

Lorsque Freud et Breuer écrivent dans les Études sur l'hystérie, l'un des premiers textes freudiens, que le trauma est un « corps étranger » occupant une "place exceptionnelle » (Ausnahmsstellung) par rapport à la psyché26, cela nous donne une indication supplémentaire sur le rapport qui doit être envisagé entre le camp et la bibliothèque : rapport non pas de reproduction ou de similarité (la bibliothèque comme signe iconique non-intentionnel $\mathrm{du} \mathrm{camp}^{27}$ ), équivalence ou équation, mais précisément rapport d'exception. Quelles en sont les implications exactes?

21 L'exception signifie la suspension provisoire de réalités en vigueur, légales ou plus généralement institutionnelles, qui continuent pourtant à être valables en principe. Cette suspension n'est donc pas une abolition des réalités, mais paradoxalement, leur confirmation. L'exception permet ainsi l'expression de deux réalités, contradictoires mais pas mutuellement exclusives. À l'ombre de la norme, l'exception tend à produire sa propre normalité et ce faisant, à devenir la règle et à se pérenniser. Giorgio Agamben a fait fructifier cette dialectique, marquée avant tout dans la pensée politico-juridique de Carl Schmitt, pour la détermination spatio-politique du camp ${ }^{28}$. Pour instaurer des camps, les national-socialistes n'eurent effectivement pas besoin de modifier l'ordre juridique existant ; au contraire, ils furent créés conformément au droit en vigueur, en invoquant la « détention préventive » ancrée dans le Code prussien (Preußisches Landrecht), applicable aux situations exceptionnelles. «Le camp, écrit même Agamben en poussant jusqu'au bout le raisonnement, est l'espace qui s'ouvre quand l'état d'exception commence à devenir la règle ${ }^{29} \%$. On pourrait ajouter, dans la perspective de la théorie du langage : le camp a pu devenir la règle parce qu'il a été d'emblée normalisé par le langage.

C'est une semblable dialectique de la norme et de l'exception qui domine apparemment le rapport entre psyché et trauma, comme le suggère en tout cas le terme de "position exceptionnelle " pour caractériser le traumatisme. Celui-ci apparaîtrait alors comme l'événement provoquant une suspension en même temps qu'une confirmation de la conscience, tout en s'instituant à l'ombre de celle-ci comme une règle insaisissable.

Dans ce contexte, on pourrait avancer que les conditions politico-juridiques permettant la création du camp correspondent aux conditions de sa mise en récit ou de son accessibilité par le souvenir. Cela permettrait tout d'abord de ne plus interpréter la rupture constatée par Levi entre les faits et leurs représentations comme une différence insurmontable, ce qui découle inévitablement d'une perspective de continuité. Dans la perspective de l'exception, cette rupture apparaîtrait bien plutôt comme un point de convergence : le camp ne peut être remémoré ni représenté, mais il fait irruption dans d'autres images, à l'intérieur desquelles il affecte d'autres images encore, en suspendant leur représentation. En contrariant ou en séparant de force la figuration ou le souvenir d'autre chose, il transparaît ex negativo: dans des lignes de rupture, des perturbations, dans le bruit de fond du discours et des récits autour de la bibliothèque.

24 Ce qu'on appréhende, c'est donc moins le camp qu'une transformation de la bibliothèque, affectée par le camp, en une institution sujette à des défaillances. Sans cesse, le symbole tant vanté de la mémoire culturelle d'une nation transparaît par moments comme un espace mémoriel traumatisé, une mémoire qui ne fonctionne pas, un espace qui déconcerte. Une mémoire qui falsifie les souvenirs, qui oublie - oubli résultant davantage 
de la déchirure des liens que de la répression des contenus - et aussi un espace au sein duquel le lecteur ne trouve pas ses repères et ressent comme une oppression physique l'insuffisance de la mémoire.

\section{Fantasme et texte littéraire}

La thèse énoncée ici est celle de la persistance d'un fantasme dans les récits sur la bibliothèque nationale de France. Il semble que, dès que cet endroit est mis en récit, un fantasme du camp commence à insister. Pourtant, rétorquerait un discours rationnel, ce fantasme ne transparaît qu'en rencontrant des lecteurs avertis de l'existence antérieure d'un camp voisin; le fantasme n'appartiendrait donc pas aux récits analysés, mais à l'imaginaire des lecteurs qui le projettent sur les discours. Or, on ne cherche pas ici à faire endosser le fantasme par quelqu'un d'autre, comme une faute. L'enjeu est bien plutôt l'émergence du fantasme comme moment de rencontre entre l'endroit réel, le récit et le lecteur. Il est décisif de ne pas se précipiter pour procéder à une séparation méticuleuse entre les éléments qui coïncident dans cette rencontre. Car l'opération rationnelle fait justement disparaître le fantasme avant même qu'il puisse être examiné plus avant.

Le défi consiste donc à extraire le fantasme de sa structure inconsciente sans le faire disparaître. Une piste allant dans ce sens est son adaptation littéraire, sa métamorphose en principe poétologique. C'est précisément là que je vois l'accomplissement de l'adaptation littéraire du motif dans Austerlitz de Sebald. Dans ce roman, le protagoniste éponyme part à la recherche de sa mémoire, disparue avec ses parents. Sa quête se déroule longtemps de manière inconsciente, ce qui entraîne sur le plan de la narration que la quasi-totalité des endroits présents parcourus par Austerlitz voient l'extermination s'inscrire comme fantasme, de manière pour ainsi dire cryptique - jusqu'au nom même de Jacques Austerlitz, écho phonétique de la métonymie usuelle du système d'extermination national-socialiste. Ce fantasme qui altère le langage empêche le souvenir, tout en le faisant reconnaître comme absolument nécessaire. Le roman naît et se développe précisément à partir de cette tension qui, même à la fin, n'est pas abolie.

Lorsque les recherches d'Austerlitz le conduisent à Paris, l'intérêt porte presque exclusivement sur le terrain situé entre la Bibliothèque nationale et la gare d'Austerlitz voisine. Cette dernière intervient en raison de son homonymie avec le protagoniste, mais aussi parce qu'elle était le point de départ des convois de déportation des Juifs parisiens.

Il est révélateur que l'exploration de la Bibliothèque nationale par Austerlitz soit précédée d'une dépression nerveuse entraînant son admission au service de psychiatrie de la Salpêtrière, rendu célèbre par Jean Charcot et situé exactement entre la bibliothèque et la gare. La lecture verticale du lieu, qui fait apparaitre une stratification bibliothèque - terrain vague - camp, est ici contrariée par une lecture horizontale de l'espace suivant un axe virtuel de contiguïté, qui produit la séquence bibliothèque - psychiatrie - gare/déportation. Par le biais de la psychiatrie, le roman fait entrer en ligne de compte le pathologique, et celui-ci apparaît même comme un facteur décisif pour révéler des liens depuis longtemps effacés au profit du «terrain vague ».

Ce qui est dit de la bibliothèque après le séjour en psychiatrie d'Austerlitz ne diffère toutefois nullement des discours de Perrault et Nora, qui ne relèvent ni de la littérature, ni de la pathologie. La bibliothèque, est-il dit dans le roman, se trouve dans un « no man's land» et «ses dimensions extérieures » aussi bien que "son agencement interne » en 
font « un endroit qui vous rebute, qui d'emblée va définitivement à contre-courant des besoins de tout véritable lecteur ${ }^{30} »$. Ajoutons d'autres syntagmes renfermant le même fantasme : gravir les marches raides «ne va pas sans danger », l'esplanade est balayée par des bourrasques de vent qui « rabattent la pluie sur ce parvis que rien n'abrite»; il est question d'un «espace [...] inoccupé », d'un "décor babylonien ». Pour accéder à leur place de travail, les lecteurs doivent être "acheminés un étage plus bas ", alors qu'ils viennent juste d'accéder avec peine au plateau. Pour Austerlitz, la descente se termine "devant une porte coulissante fermée par une chaîne» qui a l'air «de n'être que provisoire et devant laquelle des agents de sécurité en tenues ressemblant à moitié à des uniformes vous soumett[ent] à une fouille en règle ${ }^{31} »$.

Dans le jardin enserré par le rectangle des bâtiments, les arbres sont plantés comme « sur le lieu de leur exil ${ }^{32} »$. Avec les grandes baies vitrées de la salle de lecture, ils constituent un piège mortel pour les oiseaux désireux de s'y installer: «Plusieurs fois, il est également arrivé, dit Austerlitz, que des oiseaux égarés dans la forêt de la bibliothèque aient foncé sur les arbres se reflétant dans les vitres et soient tombés raides morts après un choc sourd ${ }^{33}$. " Les éléments sémantiques centraux, « rebuter », " non sans danger », " espace inoccupé ", "babylonien", " acheminer plus bas", " exil", " choc sourd", " tomber raide mort ", " accidents imprévisibles ", « chute mortelle ", renvoient moins à la bibliothèque qu'à l'endroit où les parents d'Austerlitz ont été tués. Dans le même temps, la bibliothèque s'avère totalement inutile pour Austerlitz dans sa recherche des traces laissées par son père, aperçu pour la dernière fois à Paris avant de disparaître ${ }^{34}$.

Peu après, le bibliothécaire, Lemoine, emmène Austerlitz sur le belvédère, au dixhuitième étage de la tour des Lois. Les différentes strates sur lesquelles est bâtie la bibliothèque sont alors thématisées. Le discours de Lemoine commence par les paroles déjà citées précédemment :

Sur le terrain vague où s'élève aujourd'hui cette bibliothèque, délimité par le triage de la gare d'Austerlitz et le pont de Tolbiac, il y avait par exemple jusqu'à la fin de la guerre un vaste entrepôt où les Allemands regroupaient tous les biens pillés dans les appartements des Juifs parisiens ${ }^{35}$.

Tandis que Lemoine poursuit le détail de ses explications, le soleil finit par se coucher et sous les yeux d'Austerlitz, la bibliothèque se réduit lentement à un «rectangle uniformément noir ${ }^{36}$ ». Ce "rectangle» dans lequel se fond la bibliothèque symbolise le gouffre qui attire le protagoniste ; mais par ailleurs, cette surface noire évoque également la fin de l'image, la fin des représentations et des projections. Plus de miradors, de barbelés, de portes, de chemins d'évasion. Le fantasme se retire, s'obscurcit, la sensation qui se manifeste n'a désormais plus d'objet. Imaginons Primo Levi traçant devant trente paires d'yeux une surface noire sur le tableau noir. Ou bien effaçant peu à peu son schéma du camp, laissant pour dernière image aux écoliers le rectangle noir du tableau. Dans l'acte de ce retrait de l'image se dessinent les questions, toutes les questions que la bibliothèque - «le sanctuaire de tout notre patrimoine écrit ${ }^{37}$ ", comme il est dit dans Austerlitz - laisse sans réponse. 


\section{BIBLIOGRAPHY}

Hans Günther Adler, Theresienstadt 1941-1945. Das Antlitz einer Zwangsgemeinschaft, Tübingen, Mohr, 1955.

Giorgio Agamben, Homo sacer. Il potere sovrano e la nuda vita, Turin, Einaudi, 1995 [Le pouvoir souverain et la vie nue, trad. fr. de Marilène Raiola, Paris, Seuil, 1997].

Bibliothèque nationale de France 1989-1995. Dominique Perrault, architecte, Paris, Éditions Artemis, 1995.

Josef Breuer et Sigmund Freud, Studien über Hysterie [1895], Francfort/Main, Fischer, 1991 [Études sur l'hystérie, trad. fr. d'Anne Berman, Paris, PUF, 1956].

Jean-Marc Dreyfus et Sarah Gensburger, Des camps dans Paris : Austerlitz, Lévitan, Bassano. Juillet 1943-août 1944, Paris, Fayard, 2003.

Sigmund Freud, « Die Traumdeutung » [1900], dans id., Studienausgabe, éd. par Alexander Mitscherlich, Angela Richards, James Strachey, 10 vol., t. II, Francfort/Main, Fischer, 1972 [ L'interprétation du rêve, trad. fr. de Jean-Pierre Lefebvre, Paris, Le Seuil, 2010].

Sigmund Freud, « Jenseits des Lustprinzips » [1920], dans id., Studienausgabe, éd. par Alexander Mitscherlich, Angela Richards, James Strachey, 10 vol., t. III, Francfort/Main, Fischer, 1982 [Audelà du principe du plaisir, trad. fr. de Jean Laplanche et Jean-Bertrand Pontalis, Paris, Payot, 2010]. Olivier Jacquot, « La BnF à l'emplacement d'un ancien camp nazi ? », mis en ligne en août 2010, URL http://blog.bnf.fr/lecteurs/index.php/2010/08/la-bnf-a-lemplacement-dun-ancien-campnazi/, consulté le 7 avril 2015.

Alexandra Klei, Karin Stoll et Annika Wienert (éd.), Die Transformation der Lager. Annäherungen an die Orte nationalsozialistischer Verbrechen, Bielefeld, transcript, 2011.

Serge Klarsfeld, Le calendrier de la persécution des Juifs en France 1940-1944, Paris, Les fils et filles des déportés juifs de France, The Beate Klarsfeld Foundation, 1993.

Jacques Lacan, «L'instance de la lettre dans l'inconscient ou la raison depuis Freud », dans id., Écrits I, Paris, Le Seuil, 1966.

Le Débat, $\mathrm{n}^{\circ}$ 105, mai-août 1999.

Le Débat, $\mathrm{n}^{\circ}$ 109, mars-avril 2000.

Primo Levi, I sommersi e i salvati, Turin, Einaudi, 1986 [Les Naufragés et les rescapés, trad. fr. d'André Maugé, Paris, Gallimard, 1989].

Pierre Nora, « Retour sur les lieux du crime », dans Le Débat, n 105, mai-août 1999, p. 118-121.

Dominique Perrault, «Un monument pour l'Est parisien », dans Vis-à-vis, n 2, Paris, Visualis, 1995 (numéro spécial « La Bibliothèque nationale de France »).

Charles Sanders Peirce, Collected Papers [1932], 8 vol., t. II, « Elements of Logic », éd. par Charles Hartshorne et Paul Weiss, Cambridge (Mass.), Harvard University Press, 1965 ; [ «Écrits logiques », dans id., CEuvres philosophiques, 3 vol., t. III, trad. fr. de Claudine Engel-Tiercelin et Pierre Thibaud, Paris, Le Cerf, 2006]. 
Jens Schley, Nachbar Buchenwald. Die Stadt Weimar und ihr Konzentrationslager 1937-1945, Cologne, Weimar et Vienne, Böhlau, 1999.

W[infried] G[eorg] Sebald, Austerlitz, Munich, Carl Hanser, 2001 [Austerlitz, trad. fr. de Patrick Charbonneau, Arles, Actes Sud, 2003].

Alexander Smoltczyk, « Die Türme des Schweigens », dans ZEITmagazin, n 5, 24 janvier 1997, p. 11-18 [trad. partielle : id., « Avant la Grande Bibliothèque, un grand magasin nazi. De novembre 1943 à août 1944, les biens des juifs étaient triés dans un camp de travail au 43 quai de la Gare », trad. fr. de l'agence Architexte, dans Libération, 4 février 1997, p. 18-19, URL www.liberation.fr/libe-3-metro/1997/02/04/avant-la-grande-bibliotheque-un-grand-magasinnazi-de-novembre-1943-a-aout-1944-les-biens-des-juifs-_197995, consulté le 7 avril 2015.

Wolfgang Sofsky, Die Ordnung des Terrors : Das Konzentrationslager, Francfort-sur-le-Main, Fischer, 1993 [L'organisation de la terreur - les Camps de concentration, trad. fr. d'Olivier Mannoni, Paris, Calmann-Lévy, 1995].

Nicolas Weill, «La Bibliothèque François-Mitterrand à l'ombre d'un camp nazi », dans Le Monde, 23 janvier 1997, p. 1, URL http://web.mit.edu/21f.399/unes/monde/monde0123.pdf, consulté le 7 avril 2015.

\section{NOTES}

1. W[infried] G[eorg] Sebald, Austerlitz, trad. fr. de Patrick Charbonneau, Arles, Actes Sud, 2003, p. 87.

2. Unsere Beschäftigung mit der Geschichte [ist] eine Beschäftigung mit immer schon vorgefertigten, in das Innere unserer Köpfe gravierten Bildern, auf die wir andauernd starren, während die Wahrheit irgendwo anders, in einem von keinem Menschen noch entdeckten Abseits liegt. ", voir W[infried] G[eorg] Sebald, Austerlitz, Munich, Carl Hanser, 2001, p. 105

3. Wolfgang Sofsky, Die Ordnung des Terrors: Das Konzentrationslager, Francfort/Main, Fischer, 1993, voir en particulier p. 61 et suivantes ; [trad. fr. d'Olivier Mannoni, L'organisation de la terreur - les Camps de concentration, Paris, Calmann-Lévy, 1995, p. 75 et suivantes]

4. Dans le chapitre Stereotipi [Stéréotypes] de son recueil I sommersi e i salvati [Les Naufragés et les rescapés], Levi analyse explicitement la représentation erronée du camp de concentration comme prison. Ses propos manifestent l'extension évidente de la terreur, qui dépasse largement les frontières de l'espace communément identifié avec le camp. Il écrit :

«Cette image schématique de la captivité et de l'évasion, en fait, ressemble bien peu à la situation des camps de concentration. En prenant ce terme dans son sens le plus large (c'est-àdire en y comprenant, outre les camps d'extermination aux noms connus de tout l'univers, les très nombreux camps de prisonniers et internés de guerre), il y avait en Allemagne plusieurs millions d'étrangers réduits à l'état d'esclaves, à bout de forces, méprisés, sous-alimentés, mal vêtus et mal soignés, coupés du lien avec la mère-patrie.

[...] Le cas particulier (mais numériquement imposant) des juifs était plus tragique. Même en supposant qu'ils aient réussi à franchir le réseau de barbelés et le grillage électrifié, à échapper aux patrouilles, à la surveillance des sentinelles armées de mitrailleuses sur les miradors, aux chiens dressés à la chasse à l'homme, où auraient-ils pu se diriger ? à qui demander l'hospitalité ? Ils étaient hors du monde; hommes et femmes faits de rien. Ils n'avaient plus de patrie (ils avaient été déchus de leur citoyenneté d'origine) ni de maison, mise sous séquestre au profit de citoyens de plein droit. ", id., Les Naufragés et les rescapés, trad. fr. d'André Maugé, Paris, Gallimard, 1989, p. 149 et suivantes.

[«Questa immagine schematica della prigionia e dell'evasione assomiglia assai poco alla 
situazione dei campi di concentramento. Intendendo questo termine nel suo senso più vasto (includendo cioè, oltre ai campi di distruzione dal nome universalmente noto anche i moltissimi campi per prigionieri e internati militari), esistevano in Germania parecchi milioni di stranieri in condizione di schiavitù, affaticati, disprezzati, sottoalimentati, mal vestiti e mal curati, tagliati fuori dal contatto con la madrepatria.

[...] Il caso particolare (ma numericamente imponente) degli ebrei era il più tragico. Anche ammettendo che fossero riusciti a superare lo sbarramento di filo spinato e la griglia elettrificata, a sfuggire alle pattuglie, alla sorveglianza delle sentinelle armate di mitragliatrice nelle torrette di guardia, ai cani addestrati alla caccia all'uomo: verso dove avrebbero potuto dirigersi ? a chi chiedere ospitalità ? Erano fuori del mondo, uomini e donne d'aria. Non avevano più una patria (erano stati privati della cittadinanza d'origine) né una casa, sequestrata a favore dei cittadini a pieno titolo. ", Primo Levi, I sommersi e i salvati, Turin, Einaudi, 1986, p. 124 et suivantes.]

5. Voir à ce sujet le chapitre La memoria dell'offesa [La mémoire de l'offense] dans le même volume. Levi y parle de la falsification de la mémoire par les traumatismes des blessures infligées au souvenir. Mais même si la mémoire est entraînée, si « l'exercice [...] conserve le souvenir frais et vif, [...] il est également vrai qu'un souvenir trop souvent évoqué, et exprimé sous la forme du récit, tend à se fixer en un stéréotype » (cf. Levi 1989 (note 3), p. 23 et suivante).

6. Levi 1989 (note 4), p. 153 et suivante.

7. Cf. Hans Günther Adler, Theresienstadt 1941-1945. Das Antlitz einer Zwangsgemeinschaft, Tübingen, Mohr, 1955, p. 61 [= Theresienstadt 1941-1945. Le visage d'une communauté de contrainte, non traduit, cité d'après l'édition allemande].

8. Voir les contributions dans Alexandra Klei, Karin Stoll et Annika Wienert (éd.), Die Transformation der Lager. Annäherungen an die Orte nationalsozialistischer Verbrechen, Bielefeld, transcript, 2011 [= La transformation des camps. Approches des lieux des crimes nazis, non traduit], en particulier celle des historiens Claus Kröger et Karsten Wilke sur le camp de Wewelsburg, «Konzentrationslager in der nationalsozialistischen Kriegsgesellschaft. Annäherungen an eine zweifache Ortsbestimmung ", p. 25-46 [= Les camps de concentration dans la société de guerre nazie. Approches d'une double localisation]; on lira en outre avec profit l'étude de Jens Schley, Nachbar Buchenwald. Die Stadt Weimar und ihr Konzentrationslager 1937-1945, Cologne, Weimar et Vienne, Böhlau, 1999 [= Buchenwald pour voisin. La ville de Weimar et son camp de concentration 1937-1945, non traduit].

9. Citons notamment les écrits de Primo Levi, Imre Kertész, Charlotte Delbo, Jorge Semprún, Tadeusz Borowski et Ruth Klüger, qui ne se contentent pas de témoigner de la réalité des camps, mais en problématisent également les modes de représentation.

10. Titre du documentaire d'Alain Resnais (1956) consacré à l'ancienne Bibliothèque nationale de la rue Richelieu.

11. Sebald 2003 (note 1), p. 336.

[ « Auf dem Ödland zwischen dem Rangiergelände der Gare d'Austerlitz und dem Pont Tolbiac, auf dem heute diese Bibliothek sich erhebt, war beispielsweise bis zum Kriegsende ein großes Lager, in dem die Deutschen das gesamte von ihnen aus den Wohnungen der Pariser Juden geholte Beutegut zusammenbrachten ", Sebald 2001 (note 1), p. 403]

Dans l'original allemand, le terme que P. Charbonneau traduit ici par < entrepôt > est Lager, qui désigne également le camp, notamment le camp de concentration, Konzentrationslager [note du traducteur de cet article].

12. Sur le terrain vague couvert de baraquements se trouvait, avant même la construction de la bibliothèque, une plaque commémorative rappelant l'internement de milliers de victimes du nazisme; cf. Alexander Smoltczyk, «Die Türme des Schweigens", dans ZEITmagazin, n 5, 24 janvier 1997, p. 11-18 [trad. partielle : id., « Avant la Grande Bibliothèque, un grand magasin nazi. De novembre 1943 à août 1944, les biens des juifs étaient triés dans un camp de travail au 43 quai de la Gare ", trad. fr. de l'agence Architexte, dans Libération, 4 février 1997, p. 18-19, URL 
www.liberation.fr/libe-3-metro/1997/02/04/avant-la-grande-bibliotheque-un-grand-magasinnazi-de-novembre-1943-a-aout-1944-les-biens-des-juifs-_197995]. Cette plaque a été enlevée pour la durée des travaux, mais en 2002, j'ai pu en voir moi-même une copie sur une des clôtures du chantier. Sur cette copie, la Ville de Paris s'engageait à reposer la plaque en un lieu approprié après la fin des travaux. Depuis, elle a été replacée au 43, quai Panhard-et-Levassor (anciennement 43, quai de la Gare), cf. Olivier Jacquot, «La BnF à l'emplacement d'un ancien camp nazi ? ", mis en ligne en août 2010, URL http://blog.bnf.fr/lecteurs/index.php/2010/08/labnf-a-lemplacement-dun-ancien-camp-nazi/; voir également Nicolas Weill, «La Bibliothèque François-Mitterrand à l'ombre d'un camp nazi », dans Le Monde, 23 janvier 1997, p. 1, URL http:// web.mit.edu/21f.399/unes/monde/monde0123.pdf.

13. Voir à ce sujet Serge Klarsfeld, Le calendrier de la persécution des Juifs en France 1940-1944, Paris, Association Les Fils et filles des déportés juifs de France, The Beate Klarsfeld Foundation 1993, p. 450 et suivantes et p. 849 et suivantes. On y trouve des témoignages (relativement peu nombreux) à propos des annexes du camp de Drancy sur le territoire de la ville de Paris. L'étude historique de Jean-Marc Dreyfus et Sarah Gensburger, Des camps dans Paris. Austerlitz, Lévitan, Bassano. Juillet 1943-août 1944, Paris, Fayard, 2003, est entièrement consacrée aux camps établis dans Paris intra-muros. Toutefois, les auteurs éludent la superposition spécifique du camp d'Austerlitz et de la Bibliothèque nationale. Bien plus, les deux historiens exonèrent la bibliothèque de cette contamination malsaine, accusant Sebald de falsifier l'histoire : "Sebald décrit le camp d'Austerlitz comme enseveli sous la nouvelle et pharaonique Bibliothèque nationale de France, ce qui est faux. La bibliothèque se trouve plus près de la gare d'Austerlitz, à plusieurs centaines de mètres de l'emplacement du camp. La description détaillée du camp est en total décalage avec la réalité dont elle veut témoigner. » (Dreyfus/Gensburger 2003, p. 290). La conception de l'exactitude historique exposée par Dreyfus et Gensburger immunise toutefois contre une notion de vérité qui, tout en contredisant le discours historiographique d'orientation apophantique, concerne néanmoins directement l'histoire. Leur affirmation témoigne d'une méprise radicale quant au projet sébaldien. Pour ce dernier en effet, l'enjeu n'est pas l'exactitude historique, mais la persistance en ce lieu d'un fantasme qui ne peut être chassé par la rationalisation historique.

14. Smoltczyk 1997 (note 12).

15. Smoltczyk 1997 (note 12), p.11. [«Vor einem Monat wurde mit großem Pomp die neue französische Nationalbibliothek eröffnet. Der Bauplatz sei Ödland gewesen, heißt es. In Wahrheit steht Europas größte Wissenssammlung an einem Ort der Barbarei : Schon 1943 wurden hier Bibliotheken aus jüdischem Privatbesitz konzentriert. Doch darüber spricht man nicht.»; passage non repris dans Smoltczyk 1997 Libération (note 12) et traduit spécifiquement pour le présent article.]

16. Smoltczyk 1997 Libération (note 12), p. 18, trad. complétée pour le présent article

[«Im November 1943 eröffnete die SS auf dem Eisenbahngelände Tolbiac das Camp d'Austerlitz. Es war eine Nebenstelle des Konzentrationslagers Drancy im Pariser Nordosten. [...] In unmittelbarer Nähe des heutigen Bibliotheksgeländes, am Quai de la Gare Nr. 43, wurde bis August 1944 von im Nazijargon Halb- oder mit Ariern verheirateten Juden Beutegut aus jüdischen Haushalten sortiert, um in die ausgebombten Städte des Reichs gebracht zu werden. », Smoltczyk 1997 (note 12), p. 12.]

17. Cf. Josef Breuer et Sigmund Freud, Studien über Hysterie [1895], Francfort/Main, Fischer, 1991, p. 32; [Études sur l'hystérie, trad. fr. d'Anne Berman, Paris, puf, 1956, p.5]. Le chapitre d'introduction de ce texte fondateur de la psychanalyse moderne contient des réflexions fondamentales sur la mémoire et le traumatisme. Avec les réflexions tirées de l'Interprétation du rêve de Freud et de ses études sur le langage (voir ci-dessous), elles ont plus ou moins explicitement inspiré la suite de mon propos. 
18. Cf. Sigmund Freud, «Die Traumdeutung » [1900], dans id., Studienausgabe, éd. par Alexander Mitscherlich, Angela Richards, James Strachey, 10 vol., t. ii, Francfort/Main, Fischer, 1972, p. 571 et suivantes. [L'interprétation du rêve, trad. fr. de Jean-Pierre Lefebvre, Paris, Le Seuil (Points Essais), 2010, p. 644 et suivantes].

19. Interview de Dominique Perrault, dans Bibliothèque nationale de France 1989-1995. Dominique Perrault, architecte, Paris, Éditions Artemis, 1995, p. 49 et suivantes.

20. Récemment, Laurent Matthey a soulevé cette problématique dans Building up stories. Sur l'action urbanistique à l'heure de la société du spectacle intégré, Genève, A•Type éditions, 2014.

21. Dominique Perrault, « Un monument pour l'Est parisien », dans Vis-à-vis, n 2, Paris, Visualis, 1995 (numéro spécial « La Bibliothèque nationale de France »).

22. Voir Dreyfus/Gensburger 2003 (note 13), p. 93-160.

23. Voir les dossiers consacrés à la BnF dans Le Débat, n 105, mai-août 1999, p. 118-175, et Le Débat, $\mathrm{n}^{\circ} 109$, mars-avril 2000, p. 100-137.

24. Pierre Nora, « Retour sur les lieux du crime », dans Le Débat 1999 (note 23), p. 118-121.

25. Cf. Sigmund Freud, «Jenseits des Lustprinzips » [1920], dans id., Studienausgabe, éd. par Alexander Mitscherlich, Angela Richards, James Strachey, 10 vol., t. iii, Francfort/Main, Fischer, 1982, p. 223. [Au-delà du principe du plaisir, trad. fr. de Jean Laplanche et Jean-Bertrand Pontalis, Paris, Payot, 2010, p. 49-50].

26. Cf. Breuer/Freud 1991 (note 17), p. 34 [trad. fr. : Breuer/Freud 1956 (note 17), p. 7].

27. Les icônes [icon] sont selon Peirce des signes qui renvoient à leur objet par une relation de similitude, voir Charles Sanders Peirce, Collected Papers [1932], 8 vol., t. ii, "Elements of Logic ", éd. par Charles Hartshorne et Paul Weiss, Cambridge (Mass.), Harvard University Press, 1965, p. 129-173.

28. Voir Giorgio Agamben, Homo sacer. Il potere sovrano e la nuda vita, Turin, Einaudi, 1995, notamment la troisième partie, chapitre 7 , « Il campo come nomos del moderno », p. 185-201 [ Homo sacer. Le pouvoir souverain et la vie nue, trad. fr. de Marilène Raiola, Paris, Le Seuil, 1997, III, 7, « Le camp comme « nomos > de la modernité », p. 179-193].

29. Agamben 1997 (note 28), p. 182 [orig. : «Il campo è lo spazio che si apre quando lo stato di eccezione comincia a diventare la regola », Agamben 1995 (note 28), p. 188].

30. Sebald 2003 (note 1), p. 324.

31. Sebald 2003 (note 1), p. 326.

32. Sebald 2003 (note 1), p. 328

33. Sebald 2003 (note 1), p. 328 et suivante.

34. Voir Sebald 2003 (note 1), p. 329.

35. Sebald 2003 (note 1), p. 336.

[ « Auf dem Ödland zwischen dem Rangiergelände der Gare d'Austerlitz und dem Pont Tolbiac, auf dem heute diese Bibliothek sich erhebt, war beispielsweise bis zum Kriegsende ein großes Lager, in dem die Deutschen das gesamte von ihnen aus den Wohnungen der Pariser Juden geholte Beutegut zusammenbrachten ", Sebald 2001 (note 1), p. 403]

36. Sebald 2003 (note 1), p. 338.

37. Sebald 2003 (note 1), p. 329. 


\section{ABSTRACTS}

L'essai se propose d'analyser la dimension fantasmagorique d'une superposition qui s'est créée dans l'espace urbain parisien, la Bibliothèque nationale de France (site de Tolbiac) étant construite à l'emplacement d'un ancien camp nazi. Bien que le camp nazi et la Bibliothèque soient des réalités tout à fait différentes, il y a des similitudes inquiétantes qui apparaissent grâce à cette constellation involontaire. Plusieurs discours sont pris en considération: d'abord une série de textes critiques - polémiques et éloges - sur la Bibliothèque nationale qui, d'une manière latente, comportent une mémoire inconsciente d'un camp; et ensuite un texte littéraire - le roman Austerlitz de W. G. Sebald - qui, à sa manière et avec les moyens de la fiction, met en récit la dimension spectrale de ce lieu.

Der vorliegende Essay analysiert die phantasmatische Dimension einer Überlagerung, die sich in Paris durch den Bau der neuen Pariser Nationalbibliothek am Ort eines einstigen nationalsozialistischen Lagers ergeben hat. Obwohl das Lager und die Bibliothek ganz unterschiedliche Realitäten sind, wird in dieser unwillkürlichen räumlichen Konstellation ihre beunruhigende Nähe zur Frage. Verschiedene Diskurse werden genauer untersucht : erstens eine Reihe von kritischen Texten - Polemiken ebenso wie Lobreden - über die Nationalbibliothek, die in sich auf latente Weise die unbewusste Erinnerung an ein Lager tragen; zweitens ein literarischer Text - der Roman Austerlitz von W. G. Sebald -, der auf seine Weise und mit den Mitteln der Fiktion die gespenstische Dimension dieses Ortes zu erzählen weiß.

\section{INDEX}

Chronological index: 1940, XXIe siècle

Mots-clés: trauma, histoire, bibliothèque, phantasme, terrain vague

Geographical index: Paris

\section{AUTHORS}

\section{JUDITH KASPER}

Judith Kasper enseigne la littérature française, italienne et comparée à l'université de Munich. Ses domaines de recherche concernent notamment la littérature et la Shoah, la philologie et la psychanalyse et les théories de la lecture. Sa dernière publication est Der traumatisierte Raum. Insistenz, Inschrift, Montage bei Freud, Levi, Kertész, Sebald und Dante, Berlin, New York : de Gruyter 2016. 